\title{
Angular dependence of the strong-field ionization measured in randomly oriented hydrogen molecules
}

\author{
Maia Magrakvelidze, Feng He, Sankar De, Irina Bocharova, Dipanwita Ray, Uwe Thumm, and I. V. Litvinyuk \\ Department of Physics, J.R. Macdonald Laboratory, Kansas State University, Manhattan, Kansas 66506, USA
}

\begin{abstract}
We employed electron-ion coincidence momentum spectroscopy to measure the relative angle between an emitted electron and a deuteron resulting from field dissociation of the molecular ion produced by a circularly polarized pulse. We deduced the angular dependence of the molecular ionization probability without having to align the molecules first. We determined that with $50 \mathrm{fs}$ pulses of $1850 \mathrm{~nm}$ wavelength and 2 $\times 10^{14} \mathrm{~W} / \mathrm{cm}^{2}$ intensity neutral $\mathrm{D}_{2}$ molecules are 1.15 times more likely to be ionized when the laser electric field is parallel to the molecular axis than for the perpendicular orientation, in excellent agreement with our $a b$ initio theoretical model. Our results also agree with predictions of the molecular Ammosov-Delone-Krainov (mo-ADK) theory, as well as those of a similar experiment performed with $800 \mathrm{~nm}$ pulses of comparable intensity and duration on $\mathrm{H}_{2}$ molecules.
\end{abstract}

It is now well established that strong field ionization of molecules depends critically on the angle between the molecular axes and the direction of the electric field of the laser pulse. Since tunneling field ionization is the first step of most strong-field phenomena, such as high harmonic generation $[1,2]$, nonsequential ionization $[3,4]$ and above-threshold ionization [5], it is important to know angle-differential multiphoton ionization cross sections (probabilities) to understand these phenomena quantitatively. In particular, it is important to know the angular dependence of molecular ionization in order to interpret the angle-resolved molecular high-harmonic spectra (molecular tomography [6]) as well as high-harmonic spectra associated with attosecond proton dynamics in molecules [7]. The angular dependence is determined mostly by the character of the molecule's highest occupied molecular orbital [8]. It can be measured experimentally either by using transient alignment generated by a weaker pump pulse $[9,10]$ or by determining the molecular axis direction a posteriori through measuring momenta of molecular fragments $[8,11]$. In either case it is important that the ionizing pulse did not disturb the initial angular distribution of the molecular axes. It is possible to select pulse parameters (peak intensity and duration) to satisfy this requirement for larger linear molecules and corresponding measurements were performed for $\mathrm{N}_{2}$ [8-10], $\mathrm{O}_{2}$ $[8,10], \mathrm{CO}[11], \mathrm{CO}_{2}[10,11]$, and $\mathrm{C}_{2} \mathrm{H}_{2}[11]$.

The lightest molecules $\mathrm{H}_{2}$ and $\mathrm{D}_{2}$ present a bigger challenge. In contrast to heavier molecules, it is much more difficult to precisely control their dynamical alignment due to the large separation between their rotational energy levels (low rotational inertia) and low polarizability anisotropy. Because they are so light, $\mathrm{H}_{2}$ and $\mathrm{D}_{2}$ rotate very fast when subjected to intense laser pulses and can change their orientation significantly even within the duration of very short (few cycle) laser pulses. Despite these difficulties we recently succeeded in measuring angle-dependent ionization yield of prealigned $\mathrm{D}_{2}$ molecules using $8 \mathrm{fs} 800 \mathrm{~nm}$ laser

\footnotetext{
*ivl@phys.ksu.edu
}

pulses and to put an upper limit of 1.25 on the ionization anisotropy of $\mathrm{D}_{2}$ [12]. Simultaneously, Staudte et al. [13] suggested an ingenious method for measuring the angular dependence of ionization without having to align the molecules first, based on the fact that a significant fraction of ionized hydrogen molecules dissociate (via bond softening (BS), $\left.\mathrm{H}_{2}^{+} \rightarrow \mathrm{H}^{+}+\mathrm{H}^{0}[14,15]\right)$ by the same pulse. Measuring the three-dimensional momentum of the resulting protons determines the direction of the molecular axis prior to dissociation. This, however, is not sufficient for determining the angular dependence of the ionization yield with linearly polarized pulses, since BS itself is very anisotropic and molecules were found to rotate by up to $90^{\circ}$ between ionization and dissociation events [10].

The main idea of an alignment-free experiment lies in using circularly polarized laser pulses, for which BS is still anisotropic with fragment momenta emerging mostly within a $10^{\circ}$ angle from the plane of circular polarization. However, for a rapidly rotating electric field vector there is no preferred direction within its polarization plane, and the molecular axis direction within this plane is not affected by a circularly polarized pulse. It is also important that for any detached electron the final drift momentum will coincide (up to the width of the initial electron momentum distribution) with the vector potential of the laser field at the moment of ionization. For circularly polarized pulses, the vector potential trails the rotating electric field vector by a quarter-cycle, so that ejected electrons will ultimately drift perpendicularly to the direction of the laser electric field at the moment of ionization. By measuring the distribution of relative angles within the plane of polarization between an electron and a proton coming from the same molecule, one can determine the dependence of the ionization yield on the angle between the molecule and the rotating electric field vector.

Staudte et al. [13] conducted the experiments on $\mathrm{H}_{2}$ with $40 \mathrm{fs} 800 \mathrm{~nm}$ circularly polarized pulses of varying intensities between 2 and $4.5 \times 10^{14} \mathrm{~W} / \mathrm{cm}^{2}$ and found that the experimental anisotropy decreased with increasing intensity from 1.3 to 1.18 . They also presented a simple theoretical model, which significantly overestimates the anisotropy, 
while reproducing its decrease with increasing peak intensity. It is interesting to note that the anisotropy measured by Staudte et al. gradually approaches the rate ratio (1.17) predicted by the pure tunneling molecular-ADK theory [16] at large intensity. This could simply be due to a saturation effect, since the probabilities of ionization for molecules parallel and perpendicular to laser electric field (and their ratio) approach unity with increasing intensity. A more intriguing possibility is that the ionization mechanism, and thus the observed anisotropy changes with intensity. For instance, $800 \mathrm{~nm}$ pulses of intensities in the range 1.0-2.5 $\times 10^{14} \mathrm{~W} / \mathrm{cm}^{2}$ ionize hydrogen molecules $\left(I_{p}=15.43 \mathrm{eV}\right)$ with the Keldysh parameter $\gamma$ varying from 0.7 to 1.15 . Accordingly the ionization mechanism gains in multiphoton character as the intensity decreases and the anisotropy increases.

We further investigated this issue by extending the approach of Staudte et al. to laser pulses of a longer wavelength $(1850 \mathrm{~nm})$ and using deuterium molecules as our target. In contrast to $800 \mathrm{~nm}$ wavelength, at $1850 \mathrm{~nm}$ any reasonable intensity for photoionization $\left(>10^{14} \mathrm{~W} / \mathrm{cm}^{2}\right)$ is safely within the tunneling regime (Keldysh parameter $\gamma$ $<0.5$ ). The $1850 \mathrm{~nm}$ laser pulses of $50 \mathrm{fs}$ duration were generated by a TOPAS optical parametric amplifier (Light Conversion) pumped by $1.5 \mathrm{~mJ} 35 \mathrm{fs} 800 \mathrm{~nm}$ pulses from a multipass Ti-sapphire amplifier at $1 \mathrm{kHz}$ repetition rate. After the fundamental and the signal (at $1415 \mathrm{~nm}$ ) beams were separated, the $1850 \mathrm{~nm}$ idler beam had $120 \mu \mathrm{J}$ of energy per pulse. We used a quarter wave plate (CVI Optics) to convert these pulses from linear to circular polarization. After proper attenuation the resulting beam was focused by a $75 \mathrm{~mm}$ focal length spherical on-axis mirror onto a supersonic jet of deuterium molecules in the interaction region of a COLTRIMS apparatus. The molecular jet and the laser beam were perpendicular to each other and both normal to the axis of a uniform electric field $(10 \mathrm{~V} / \mathrm{cm})$ spectrometer terminated on both sides by time- and position-sensitive delay-line anode detectors (Roendtek) for measuring electron and ion momenta in coincidence. A magnetic field of $13 \mathrm{G}$ parallel to the spectrometer axis was employed to contain the electrons and supplemented by a smaller perpendicular magnetic field to compensate for the Earth's magnetic field. The gas target density was adjusted so that much less than one charged fragment of each kind was detected per laser pulse. The peak intensity of the laser pulses was determined in situ by measuring momentum distributions of intact $\mathrm{D}_{2}^{+}$ions as described in $[9,17]$.

As described above, we measured the relative angle (in the plane of the circular polarization of our laser pulses) between an emitted electron and a $\mathrm{D}^{+}$ion resulting from a single event. Figure 1 presents the kinetic energy and twodimensional momentum spectra for $\mathrm{D}^{+}$ions. At our experimental intensity the spectrum includes two distinct energy bands. The low energy band is due to the one-photon BS and the net two-photon above-threshold dissociation (ATD, [18]) processes. The BS and ATD bands are not resolved and in our analysis we considered them together. Double ionization (Coulomb explosion) appears as a broad peak at $2.6 \mathrm{eV}$. Since we are measuring the angular dependence of the single ionization process, we were only interested in the low energy

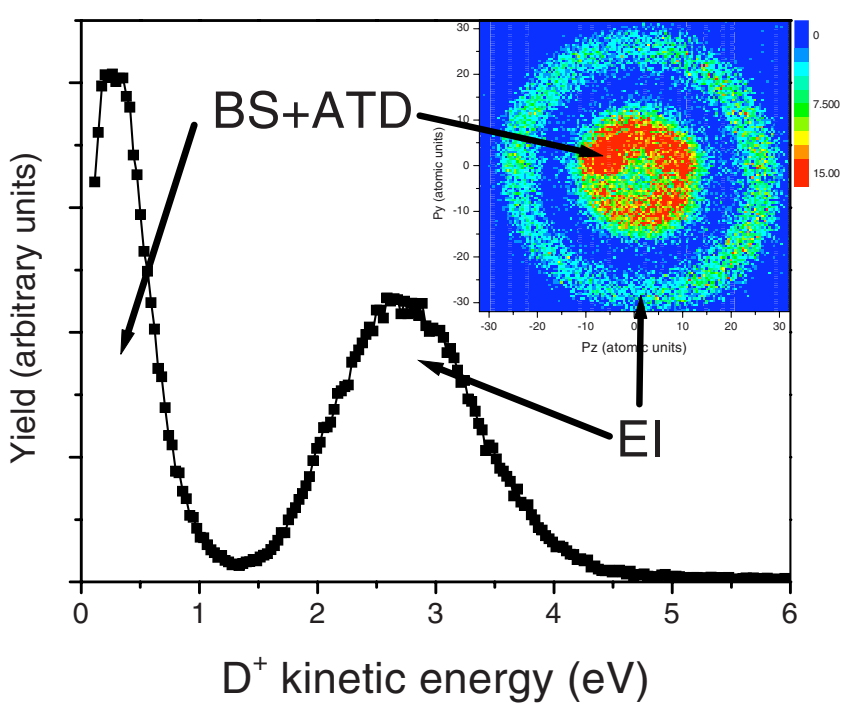

FIG. 1. (Color online) Kinetic energy and two-dimensional (2D) momentum (inset) spectra of $\mathrm{D}^{+}$fragments generated by circularly polarized laser pulses of $1850 \mathrm{~nm}$ wavelength, $50 \mathrm{fs}$ duration, and $4 \times 10^{14} \mathrm{~W} / \mathrm{cm}^{2}$ peak intensity. The low energy band (smaller circle in momentum spectrum) is due to bond softening (BS) and above-threshold dissociation (ATD). The high energy band is due to the enhanced ionization (EI) at the critical distance.

$(0-1 \mathrm{eV})$ deuterons resulting from dissociation of the molecular ions. Not being able to detect the accompanying neutral $\mathrm{D}^{0}$ atomic fragment, we must rely on the low overall count rate to ensure that both charged particles come from the same molecule. The experimental distribution of the relative angles between the ion and electron momenta is shown in Fig. 2. One can see that there is a slight anisotropy in that distribution with more electrons detected in direction perpendicular to the molecular axis. Since the ultimate drift velocity of a photoelectron is perpendicular to the instantaneous direction of the electric field vector at the moment of ionization, we conclude that more molecules are ionized when their axes are parallel than when they are normal to the field. The ratio of relative electron yields detected at angles of $90^{\circ}$ and $0^{\circ}$ to the molecular axis is equal to the ratio of ionization probabilities for molecules aligned parallel and perpendicular to linearly polarized electric fields of the same field strengths. In our case the electric field amplitude in circularly polarized pulses of $4 \times 10^{14} \mathrm{~W} / \mathrm{cm}^{2}$ peak intensity is the same as for $2 \times 10^{14} \mathrm{~W} / \mathrm{cm}^{2}$ linearly polarized pulses. We determine that for such pulses the ratio of maximum and minimum ionization probabilities (corresponding to parallel and perpendicular alignments, respectively) is $1.15( \pm 0.05)$. This is in good agreement with values reported by Staudte et al. for $\mathrm{H}_{2}$ with $800 \mathrm{~nm}$ pulses of comparable intensity.

Our value also agrees well with the result of the quasistatic molecular-ADK theory which assumes pure tunneling ionization [16]. However, other theoretical models vary in their predictions $[13,19]$. In particular, Staudte et al. presented a modified molecular strong-field approximation model which overestimates the ionization anisotropy of their experimental results. In view of this discrepancy, we developed a separate wave-function propagation model $[15,20]$ for calculating ionization probabilities as a function of the angle 

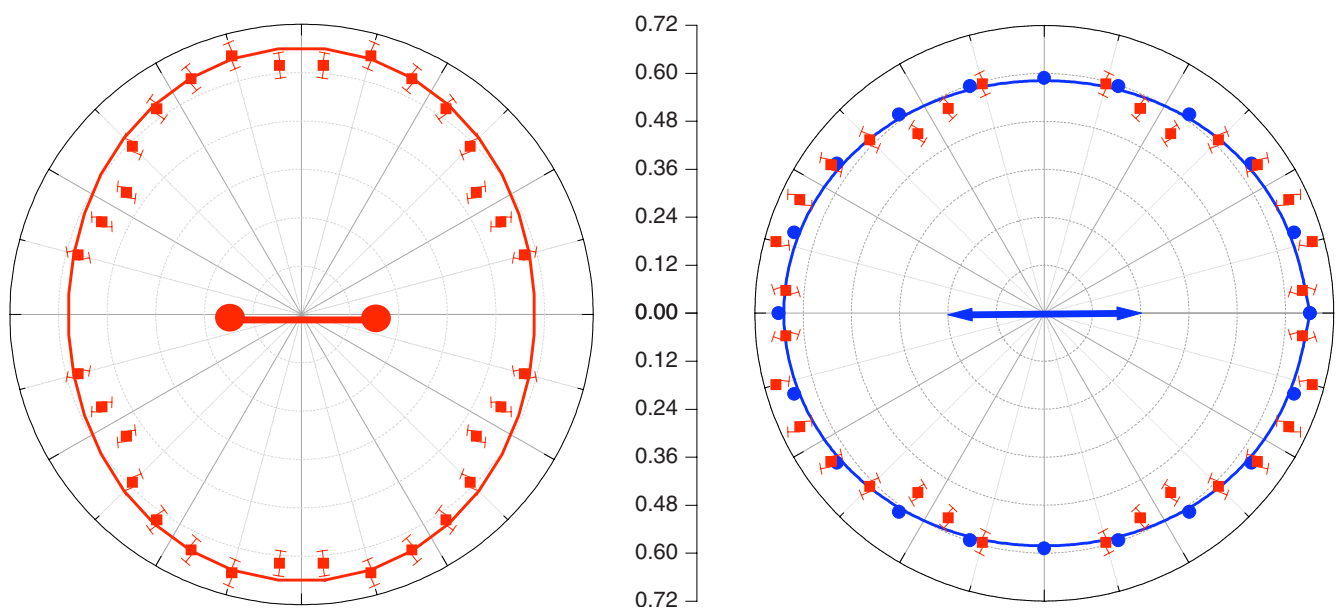

FIG. 2. (Color online) Left: experimental distribution of relative angles between momenta of low energy $(0-1 \mathrm{eV}) \mathrm{D}^{+}$ions and coincident electrons generated by circularly polarized laser pulses of $1850 \mathrm{~nm}$ wavelength, 50 fs duration, and $4 \times 10^{14} \mathrm{~W} / \mathrm{cm}^{2}$ peak intensity. The solid line shows the best fit of the data by the function $\mathrm{A}\left(\cos ^{2} \theta+\varepsilon \sin ^{2} \theta\right)$, where $\varepsilon=1.15 \pm 0.05$ represents the ratio of coincident fragment and photoelectron yields at $90^{\circ}$ and $0^{\circ}$ angles. Right: calculated single ionization probability for $\mathrm{D}_{2}$ as a function of the angle between the molecular axis and electric field vector of the corresponding linearly polarized pulse of $2 \times 10^{14} \mathrm{~W} / \mathrm{cm}^{2}$ peak intensity (blue circles); the solid line shows the best fit with anisotropy $\varepsilon=1.12$; red squares correspond to the same data as on the left, scaled and rotated by $90^{\circ}$.

between the molecular axis and the electric field of a linearly polarized laser pulse. In this model we use the single active electron approximation to solve the time-dependent twodimensional Schrödinger equation (TDSE) in the velocity gauge in Cartesian coordinates (we use atomic units, $e=m$ $=\hbar=1$, unless stated otherwise),

$$
i \frac{\partial}{\partial t} \Phi(x, y ; t)=\left[\frac{P_{x}^{2}+P_{y}^{2}}{2}-\frac{P_{x} A(t)}{c}+V(x, y)\right] \Phi(x, y ; t) .
$$

The electron-nuclei interaction is modeled in terms of the soft-core Coulomb potential

$$
V(x, y)=-\sum_{j= \pm 1} \frac{z_{\mathrm{eff}}}{\sqrt{\left(x+j \frac{R_{0}}{2} \cos \theta\right)^{2}+\left(y+j \frac{R_{0}}{2} \sin \theta\right)^{2}+\alpha}},
$$

where $x$ and $y$ are the electron coordinates in the plane of laser polarization and internuclear axis; and $P_{x}$ and $P_{y}$ are the momentum components in $x$ and $y$ directions. The internuclear distance is kept fixed at $R_{0}=1.4$, corresponding to the equilibrium distance for the ground state of $\mathrm{D}_{2}$. The electric laser field in our calculations points along the $x$ axis. $\theta$ is the angle between the laser polarization and the internuclear axis. For a soft-core parameter $\alpha=0.64$ and an effective nuclear charge $z_{\text {eff }}=0.58$ our single active electron model reproduces the ionization potential both at equilibrium internuclear distance and at infinite separation (with $z_{\text {eff }}=1$ ). We use the split-operator Crank-Nicholson propagation scheme to solve the TDSE with a time step of 0.1. The numerical grid has 2000 and 800 points in the $x$ and $y$ directions, respectively, with the spatial steps $\Delta x=\Delta y=0.3$. The vector potential $\boldsymbol{A}$ is associated with the laser electric field,

$$
E(t)=E_{0} \sin \omega t \sin ^{2}(\pi t / \tau)=-\frac{\partial}{\partial t} A(t)
$$

with amplitude $E_{0}$ (directed along the $x$ axis), angular frequency $\omega$, and duration $\tau$. We apply a $\cos ^{1 / 8}$ masking function over a length of 60 (30) from the ends of the rectangular numerical grid in $x(y)$ direction in order to suppress unphysical reflections. We calculate the ionization probability as $1-P_{\text {bound }}$, where $P_{\text {bound }}$ is the probability for the electron

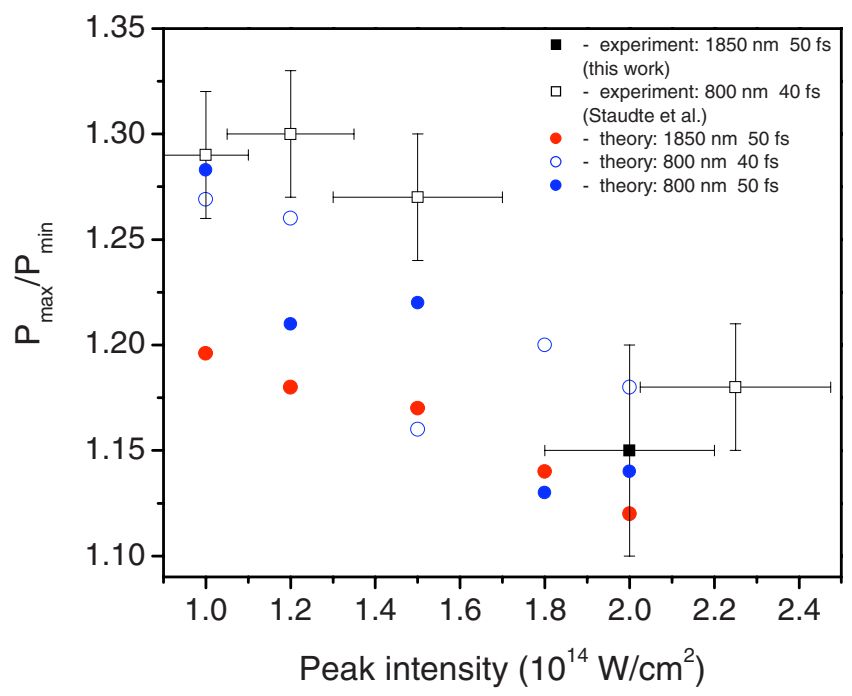

FIG. 3. (Color online) Angular anisotropy of single ionization of $\mathrm{D}_{2}$ at various peak intensities and pulse durations calculated for $1850 \mathrm{~nm}$ (solid red circles, $50 \mathrm{fs}$ ) and $800 \mathrm{~nm}$ (hollow blue circles $-40 \mathrm{fs}$; solid blue circles $-50 \mathrm{fs}$ ) pulses. Experimental results from this paper for $1850 \mathrm{~nm}$ (solid square) and from Staudte et al. for $800 \mathrm{~nm}$ (hollow squares) are shown for comparison. The intensities for experimental data are reduced by a factor of 2 to make the electric field values the same as in the calculations. 
to remain inside the propagation area of the numerical grid at the end of the calculation. In order to ensure converged results, we propagate the wave function past the laser pulse until the ionization probability has stabilized.

Figure 2 shows the calculated ionization rate as a function of the angle $\theta$ for a laser intensity, wavelength, and pulse duration of $2 \times 10^{14} \mathrm{~W} / \mathrm{cm}^{2}, 1850 \mathrm{~nm}$, and $50 \mathrm{fs}$ (FWHM), respectively. As expected, molecules aligned parallel to the laser polarization are easier to ionize than those aligned perpendicularly. The ratio of the maximum and the minimum ionization probabilities is 1.12 , which is in excellent agreement with our experimental value of $1.15 \pm 0.05$. Figure 3 presents the intensity dependence of the ionization anisotropy for hydrogen molecules at 800 and $1850 \mathrm{~nm}$ wavelengths, with pulse lengths of 40 and $50 \mathrm{fs,} \mathrm{where} \mathrm{experi-}$ mental results of Staudte et al. are also shown for comparison. Overall our simulation underestimates the experimental values very slightly. This small discrepancy could be explained by the fact that the calculations were performed for a single intensity, taken to be the peak intensity of the pulse, while in the experiment a range of intensities contributes to the final anisotropy due to temporal spatial variations within the pulse. Since the anisotropy is higher for lower intensities, single intensity calculations are expected to un- derestimate its value. Deviations in the intensity dependence of the anisotropy at $800 \mathrm{~nm}$ from a monotonous decrease are consistent with a numerical uncertainty in our calculated ionization probabilities of $0.3 \%$.

To summarize, we measured the angular dependence of the strong-field ionization probability for $\mathrm{D}_{2}$ in $1850 \mathrm{~nm}$ infrared laser pulses. We determined that this angular dependence exhibits a weak anisotropy with an ionization yield ratio of $1.15 \pm 0.05$ favoring the ionization of molecules that are aligned parallel to the electric field. We also presented a 2D single active electron $a b$ initio numerical model which accurately predicts the measured anisotropy, as well as its intensity dependence for both our experiment at $1850 \mathrm{~nm}$ and Staudte et al.'s at $800 \mathrm{~nm}$ wavelength. Both our experimental and theoretical values agree very well with the results of Staudte et al. for $\mathrm{H}_{2}$, as well as with our own earlier estimate for $\mathrm{D}_{2}$ obtained using dynamic alignment and fewcycle 800 fs pulses.

This work was supported by funding from Chemical Sciences, Geosciences and Biosciences Division, Office of Basic Energy Sciences, Office of Science, U.S. Department of Energy and the NSF.
[1] K. S. Budil, P. Salieres, A. LHuillier, T. Ditmire, and M. D. Perry, Phys. Rev. A 48, R3437 (1993).

[2] I. Mercer, E. Mevel, R. Zerne, A. LHuillier, P. Antoine, and C. G. Wahlstrom, Phys. Rev. Lett. 77, 1731 (1996).

[3] D. N. Fittinghoff, P. R. Bolton, B. Chang, and K. C. Kulander, Phys. Rev. Lett. 69, 2642 (1992).

[4] B. Walker, E. Mevel, B. Yang, P. Breger, J. P. Chambaret, A. Antonetti, L. F. DiMauro, and P. Agostini, Phys. Rev. A 48, R894 (1993).

[5] G. G. Paulus, F. Grasbon, A. Dreischuh, H. Walther, R. Kopold, and W. Becker, Phys. Rev. Lett. 84, 3791 (2000).

[6] J. Itatani, J. Levesque, D. Zeidler, H. Niikura, H. Pepin, J. C. Kieffer, P. B. Corkum, and D. M. Villeneuve, Nature (London) 432, 867 (2004).

[7] S. Baker, J. S. Robinson, C. A. Haworth, H. Teng, R. A. Smith, C. C. Chiril, M. Lein, J. W. G. Tisch, and J. P. Marangos, Science 312, 424 (2006).

[8] A. S. Alnaser, S. Voss, X. M. Tong, C. M. Maharjan, P. Ranitovic, B. Ulrich, T. Osipov, B. Shan, Z. Chang, and C. L. Cocke, Phys. Rev. Lett. 93, 113003 (2004).

[9] I. V. Litvinyuk, K. F. Lee, P. W. Dooley, D. M. Rayner, D. M. Villeneuve, and P. B. Corkum, Phys. Rev. Lett. 90, 233003 (2003).

[10] D. Pavičić, K. F. Lee, D. M. Rayner, P. B. Corkum, and D. M. Villeneuve, Phys. Rev. Lett. 98, 243001 (2007).
[11] A. S. Alnaser, C. M. Maharjan, X. M. Tong, B. Ulrich, P. Ranitovic, B. Shan, Z. Chang, C. D. Lin, C. L. Cocke, and I. V. Litvinyuk, Phys. Rev. A 71, 031403(R) (2005).

[12] I. A. Bocharova, H. Mashiko, M. Magrakvelidze, D. Ray, P. Ranitovic, C. L. Cocke, and I. V. Litvinyuk, Phys. Rev. A 77, 053407 (2008).

[13] A. Staudte, S. Patchkovskii, D. Pavičić, H. Akagi, O. Smirnova, D. Zeidler, M. Meckel, D. M. Villeneuve, R. Dörner, M. Y. Ivanov, and P. B. Corkum, Phys. Rev. Lett. 102, 033004 (2009).

[14] P. H. Bucksbaum, A. Zavriyev, H. G. Muller, and D. W. Schumacher, Phys. Rev. Lett. 64, 1883 (1990).

[15] B. Feuerstein and U. Thumm, Phys. Rev. A 67, 043405 (2003); U. Thumm, T. Niederhausen, B. Feuerstein, ibid. 77, 063401 (2008).

[16] X. M. Tong, Z. X. Zhao, and C. D. Lin, Phys. Rev. A 66, 033402 (2002).

[17] A. S. Alnaser, X. M. Tong, T. Osipov, S. Voss, C. M. Maharjan, B. Shan, Z. Chang, and C. L. Cocke, Phys. Rev. A 70, 023413 (2004).

[18] B. Yang, M. Saeed, L. F. DiMauro, A. Zavriyev, and P. H. Bucksbaum, Phys. Rev. A 44, R1458 (1991).

[19] A. Apalategui and A. Saenz, J. Phys. B 35, 1909 (2002).

[20] F. He, A. Becker, and U. Thumm, Phys. Rev. Lett. 101, 213002 (2008). 